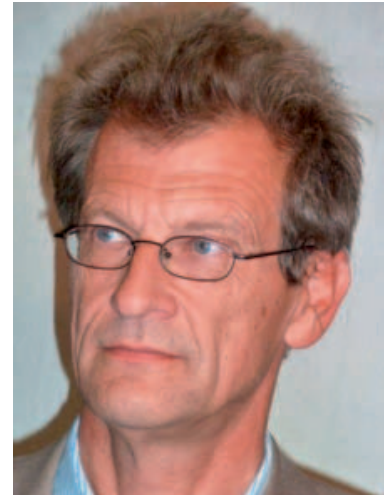

Dr. med. Dr. rer. nat. Bernhard Uehleke, Berlin, Deutschland

\section{Komplementärmedizin in Indien - Eindrücke einer Reise}

Indien besteht aus 29 Bundesstaaten und hat rund eine Milliarde Einwohner. Bewundernswert ist hierbei, wie so viele Menschen mit verschiedenstem Hintergrund und Einkommen sowie mit unterschiedlichen Religionen im Grossen und Ganzen friedlich in einem demokratischen System zusammenleben. Insgesamt kommt eine Reise nach Indien meist einer Zeitreise gleich: An vielen Stellen herrschen Zustände wie in Zentraleuropa vor 20, 50 oder gar 100 Jahren. Allerdings gibt es auch einige Bezirke in den Grossstädten, die geradezu futuristisch erscheinen; so ist z.B. der neue und gut funktionierende Flughafen in NeuDelhi an eine topmoderne U-Bahn angeschlossen, die einen rasch und effektiv ins Zentrum befördert. Unmittelbar neben dem Hauptbahnhof in Delhi gibt es den «Markt» mit einem Chaos an Geschäften und Ständen, die durchaus ins Mittelalter passen könnten, wären da nicht die vielen Motorräder, Autos und Rikschas sowie die überall in einem oberflächlichen Kabelgewirr präsente Elektrifizierung, wobei ständige und mitunter tagelang anhaltende Stromausfälle fast überall in Indien an der Tagesordnung sind. Das gesamte Geschäftsleben von Indien scheint ausschliesslich per Mobiltelefon abgewickelt zu werden, während Papierdokumente oder E-Mails weitgehend überflüssig sind - ein Zustand, der Europäer überraschen mag. Viele Menschen können nicht schreiben und lesen, und für einfachste Rechenaufgaben wird immer ein Taschenrechner benötigt. Nur 30\% der Bevölkerung sollen Zugang zu einer Toilette haben; dementsprechend sind Infektionen wie z.B. Ruhr und insbesondere Typhus weit verbreitet, vor allem während der Monsunzeit.

Genauso weit gefächert wie das Land mit seinen sozialen und religiösen Gegensätzen ist die medizinische Versorgung. Ja, es gibt Hightechkliniken und private Zentren auf hohem westlichem Standard für die Reichen (und Touristen). Manche Westler kommen extra nach Indien, weil hier etwa eine kosmetische Operation doch noch deutlich preiswerter ist. Eine «Clinic» auf dem Lande entspricht einer westlichen Praxis aus dem Anfang des 19. Jahrhunderts. Der «Doctor» sitzt mit seinem Patienten in einem winzigen Untersuchungsraum, in den nicht einmal eine Untersuchungsliege passen würde, und hat ausser seinem Stethoskop und seinem Schreibstift keine weiteren Utensilien; bestenfalls gibt es noch eine Möglichkeit zur Blutentnahme, und oft findet sich noch ein altes Mikroskop. Bezüglich der Vertraulichkeit stellt man sich nicht so an, sodass meist nicht nur Freunde und Familienmitglieder des Patienten im Behandlungsraum oder Vorzimmer herumstehen - die Türen sind ja sowieso meist offen.

Ayurvedische und homöopathische Arztpraxen sehen häufig noch bescheidener aus. Bei manchem AyurvedaArzt sieht es vorne wie in einem Gewürzladen aus, während dahinter in einer vielleicht nur mit einem Laken etwas abgetrennten Ecke dann der Doktor sitzt (Abb. 1), und manche Zahnarztpraxis auf dem Lande unterscheidet sich kaum vom Friseur. Die Apotheken sind manchmal nur in einem Kiosk auf $3 \mathrm{~m}^{2}$ untergebracht, bieten aber dennoch eine erstaunliche Auswahl. Dort ebenso wie in den grossen Apotheken in den Städten werden Tabletten überwiegend

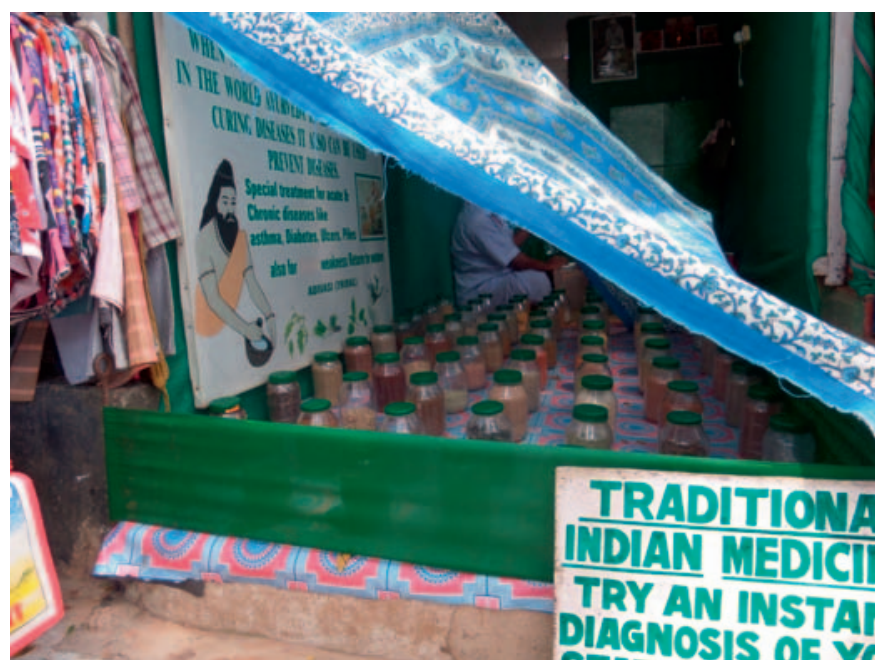

Abb. 1. Einblick in eine indische Ayurveda-Praxis.

\section{KARGER}

Fax +497614520714

Information@Karger.com

www.karger.com (c) 2015 S. Karger GmbH, Freiburg

1015-0684/15/0274-0196\$39.50/0
Dr. med. Dr. rer. nat. Bernhard Uehleke

Karwendelstrasse 13, 12203 Berlin, Deutschland

b.uehleke@gmx.net 


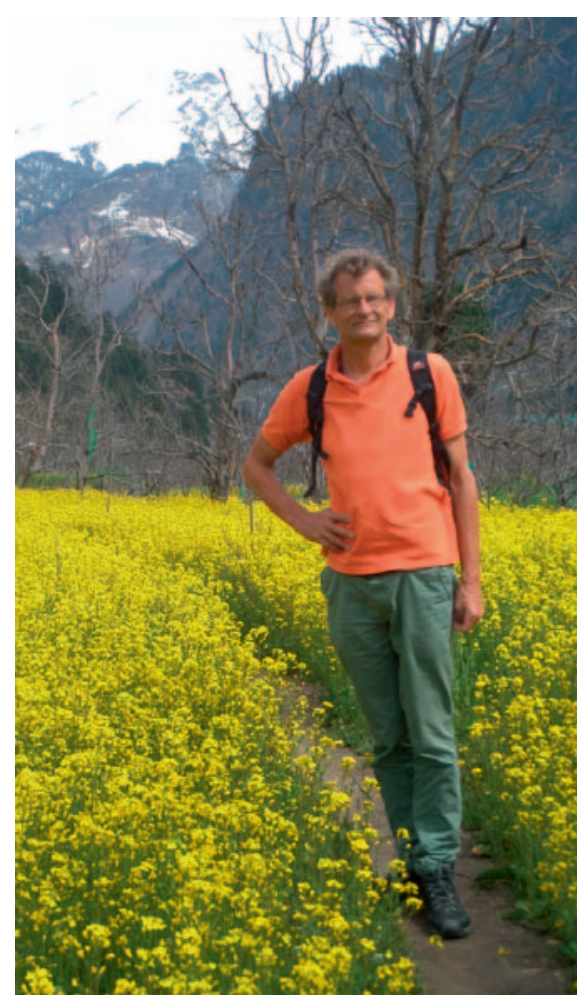

stückweise oder blisterweise aus Klinikpackungen verkauft - dann natürlich ohne jegliche Patienteninformation. Auf Nachfrage hin verweist dann der Apotheker auf das Internet und ist oft dabei behilflich. Als Westler erhält man vieles auch ohne Rezept und alles unglaublich billig.

Wenn man den ayurvedischen Doktor vor Augen hat, wird man davon überrascht, dass die Ausbildung heute erstaunlich umfassend und vom Staat geregelt ist. Es besteht offiziell ein duales medizinisches System mit (Western) Medicine auf der einen und Traditional Indian Medicine auf der anderen Seite. Letztere umfasst neben Ayurveda und Unani (im indischen Subkontinent gebräuchlicher Begriff für die graeco-arabische Medizin) auch die Homöopathie. Dies wird damit begründet, dass die Homöopathie schon fast 200 Jahre lang in Indien eine gewisse Rolle spielt. Im Gesundheitsministerium gibt es eine eigene Hauptabteilung, d.h. Ayurveda, Yoga and Naturopathy, Unani, Siddha and Homoeopathy (AYUSH), für diese komplementärmedizinischen Richtungen. Das Studium für einen Therapeuten in einer dieser Richtungen kann an rund 160 speziellen Hochschulen absolviert werden. Das Curriculum für den «Dr. Ay» stellt im Prinzip ein gestrafftes Medizinstudium einschliesslich aller möglichen Grundlagenfächer dar, hinzu kommen umfangreiche Quellenstudien und das Lernen der Ayurvedischen Praxis. Da Heilkräuter in der ayurvedischen Therapie eine recht grosse Rolle spielen und diese ja direkt vom ayurvedischen Doktor mitverkauft werden, wird die Kräuterheilkunde entsprechend vertieft vermittelt und dürfte dem Lehrgebiet «Pharmazeutische Biologie» des europäischen Pharmaziestudiums in etwa entsprechen. Die indische Regierung ist derzeit daran interessiert, die Ayurvedische Medizin zu stärken und international zu verbreiten - ähnlich wie es China mit der Traditionellen Chinesischen Medizin bzw. Akupunktur gelungen ist. Die Diskussionen und Überlegungen, die auch unter Hinzunahme von Ayurveda-Spezialisten aus anderen Ländern derzeit geführt bzw. angestellt werden, ähneln in erschreckender Weise der Situation, die in Deutschland beispielsweise in den 1980er Jahren üblich war. Viele Anhänger des Ayurveda (auch so mancher prominenter Professor an einem der Ayurvedischen Colleges) halten eine Erforschung der Wirksamkeit mittels klinischer Studien aus verschiedenen Gründen schon grundsätzlich für unmöglich oder kontraproduktiv! Es gibt aber in Indien leider kaum Erfahrungen mit klinischen Prüfungen über Ayurveda; die wenigen publizierten klinischen Studien weisen erhebliche Mängel auf. Da die akademischen Welten zwischen den Hochschulen für Westliche Medizin und für Ayurvedische Medizin recht voneinander abgetrennt $\mathrm{zu}$ sein scheinen, ist es bisher nicht üblich, das dringend benötigte Know-how zur Planung und Durchführung einer klinischen Studie aus den entsprechenden Fächern (klinische Pharmakologie, medizinische Biometrie usw.) der besser ausgestatteten Hochschulen für Westliche Medizin «anzuzapfen».

Für die Inder ist die Westliche Medizin weitaus attraktiver, jedoch wird oft aus Gründen der Verfügbarkeit und der Kosten eher widerstrebend auf den Ayurveda-Arzt ausgewichen. Viele der Absolventen aus den Colleges finden kein Einkommen. Sie werden mitunter in staatliche Versorgungsprogramme eingebunden, z.B. in die nun eingerichteten stationären Geburtseinrichtungen. Dazu erhalten sie eine siebenmonatige Spezialausbildung in Geburtshilfe und werden dann ergänzend zu den Fachärzten in den Dienstplan eingeteilt.

Paradoxerweise kommen die Ayuveda-Kuren heutzutage vor allem zur Entschlackung und zur Entgiftung bei übergewichtigen Menschen aus dem Westen zum Einsatz, während in Indien selbst oft unterernährte Menschen, insbesondere Frauen und auch Kinder, anzutreffen sind. Bei den ausländischen Klienten kommt dann noch mancherlei esoterischer/spiritueller Aberglauben hinzu, der manchem eine Kur bei einem Guru in Indien als überlegen gegenüber dem westlichen Heilfasten erscheinen lassen mag ... 